\title{
WEB 2.0, COMUNICACIÓN Y MATERIAL DIDÁCTICO DIGITAL PARA EL APRENDIZAJE DEL ESPAÑOL: EL AULA VIRTUAL DE ESPAÑOL DEL INSTITUTO CERVANTES Y SU ACTUALIZACIÓN
}

\author{
WEB 2.0, COMMUNICATION AND DIDACTIC DIGITAL \\ E-RESOURCES FOR LEARNING SPANISH: THE STATE OF ART OF \\ THE AULA VIRTUAL DE ESPAÑOL AT INSTITUTO CERVANTES
}

\author{
OLGA JUAN LÁZARO \\ Instituto Cervantes, Madrid. España. \\ olgajuan@cervantes.es
}

\section{RESUMEN}

Las Tecnologías de la Información y la Comunicación han irrumpido con la Web Social o Web 2.0 en el ámbito educativo cuando todavía estamos reflexionando e integrando modelos que incorporan la tecnología en el aula, y desarrollando los modelos de enseñanza semipresencial y a distancia en el aprendizaje de lenguas. Desde el Instituto Cervantes se ha creado el Aula Virtual de Español, una plataforma de enseñanza-aprendizaje con cursos de español general y cursos para la formación de profesores. En este artículo revisamos los planteamientos y cambios que implica la Web Social para proponer y justificar la evolución del AVE al AVE SOCIAL; repasamos la actual propuesta respecto al modelo de integración del MDD (Material Didáctico Digital) en el aula presencial promoviendo la autonomía del estudiante; y describimos la experiencia de formación de tutores del AVE a distancia siguiendo un enfoque socioconstructivista del aprendizaje en línea.

Palabras clave: Web social, TIC (Tecnologías de la Información y la Comunicación), enseñanza a distancia, enseñanza semipresencial, aprendizaje de español, cursos de español en Internet, formación de profesores online.

\section{ABSTRACT}

The Information and Communication Technologies irrupted with the Social Web or Web 2.0 in the educational branch, when we were still thinking about the effective way to integrate the ICT in the classroom, and developing the models of blended-learning and e-learning for foreign languages. The Instituto Cervantes has conceived and design the Aula Virtual de Español as a communication environment in which the student has access to the Spanish Courses and the teachers to the online tutor course. In this article, we 
review the Social Web paradigm, to justify the evolution from the AVE to the Social AVE. We propose five stages to use the e-resources in the classroom promoting the student's autonomy and we describe the e-learning experience teaching future tutors of the AVE following the socioconstructivism paradigm.

Keywords: Social web, ICT (Information and Communication Technologies), distance learning, Spanish as foreign language, Spanish online courses, e-learning, online tutors.

Recibido: 02-08-2009. Aceptado: 19-10-2009.

\section{INTRODUCCIÓN}

Ceguir los pasos de los avances en Tecnologías de la Información y la Comuni$\checkmark$ cación, las TIC, teniendo como objetivo la difusión de la lengua y la cultura hispana, implica no sólo la inversión en una gestión tecnológica en constante evolución y cambio, sino una inversión en la gestión de contenidos, su conceptualización, su estructura, su diseño, etc., encaminados siempre a conseguir los objetivos propuestos. En el caso de la enseñanza de la lengua y la cultura, la apuesta por las TIC conllevan la experimentación de nuevos modelos de aprendizaje, una reflexión constante en la que estamos implicados no sólo estudiantes y profesores de los más de 70 centros que tiene el Instituto Cervantes, sino, además de la Dirección Académica, toda la estructura orgánica de la institución debido a las implicaciones administrativas, presupuestarias, reorganizativas que significa la integración de la tecnología en el aula y fuera de ella, con su reorientación metodológica y con la experimentación en modelos de aprendizaje a distancia tutorizados.

En este artículo vamos a dedicar el apartado 2 a comentar las últimas novedades que desde el punto de vista tecnológico se han desarrollado en el Instituto, así como analizaremos algunas de las páginas más visitadas del Centro Virtual Cervantes por profesores de ELE (español lengua extranjera) y EL2 (español como segunda lengua).

Esta presentación nos va a dar pie a centrarnos en el epígrafe 3 en el Aula Virtual de Español, AVE, como eje en torno al que se articula el desarrollo tecnológico para el aprendizaje y la enseñanza del español en el Instituto, y en torno al cual estamos definiendo y experimentando los cambios en los modelos de enseñanza que incorporan las TIC.

El capítulo 4 es el más extenso dado que es en el que describimos y argumentamos desde diferentes puntos de vista lo que es la Web Social orientada a su repercusión y aceptación en la educación, siempre de forma concisa para atenernos a la extensión prevista para el artículo. Esta revisión del estado de la cuestión es la que nos lleva a tomar unas primeras decisiones sobre las fases de actualización del AVE para convertirse en el AVE SOCIAL, a la vez que se abren otra serie de premisas 
que tendremos que ir respondiendo en paralelo a través de la comunicación con los equipos docentes del Instituto y con los de las instituciones a nivel internacional que han optado por incorporar el AVE a su actividad académica en la enseñanza del español, sin olvidar la consultoría con equipos expertos. El debate y el diálogo son constantes e imprescindibles en el procedimiento de trabajo de un proyecto de estas dimensiones, de forma que se pueda diseñar un proceso evolutivo que dé respuesta a las necesidades que se plantean a los usuarios del sistema.

La enseñanza presencial, es decir, el aula, ha venido siendo hasta ahora una práctica cultural habitual. La integración de las TIC en la educación ha ido perfilando una realidad compleja en la que había que tener en cuenta todos los factores que articulan la gestión de un centro (debido a la dotación informática, por ejemplo), además de los metodológicos y la propia generación de contenidos adaptados al currículum. Esto nos ha llevado a centrarnos en el apartado 5 en los Materiales Didácticos Digitales dentro de la plataforma AVE, abordándolos desde la perspectiva del desarrollo de la autonomía que va a propiciar en los estudiantes, gracias a aspectos que favorecen la accesibilidad de los recursos puestos a su alcance a través de una conexión a Internet así como a la estabilidad que se garantiza desde el Instituto Cervantes de toda la plataforma en servidores de alta disponibilidad.

Por último, no queríamos terminar estas reflexiones sin mencionar el constructivismo en el aprendizaje a distancia. Hemos ejemplificado esta visión desde nuestra experiencia en los cursos a distancia de formación de tutores del AVE, que suman ya tres convocatorias, donde los tutores valoran especialmente el trabajo cooperativo promovido entre los aprendientes, a partir de dinámicas y propuestas de actividades diseñadas por los profesores de ELE en la propia plataforma AVE.

\section{EL INSTITUTO CERVANTES Y LA APUESTA TECNOLÓGICA PARA LA PROMOCIÓN DE LA LENGUA Y CULTURA HISPANAS}

Desde el primer momento de su creación, el Instituto Cervantes ${ }^{1}$ ha apostado por la inclusión de la tecnología en todos los ámbitos del desempeño de sus funciones, dando curso de la manera más efectiva posible a uno de los objetivos fundacionales, la promoción de la lengua y la cultura españolas. De esta forma, se investiga en la calidad de los servicios y productos ofrecidos por el Instituto Cervantes y se invierte en la creación y actualización constante de los mismos. A modo de ejemplo, se ha puesto a disposición del público la Isla Cervantes en Second Life en http://secondlife.cervantes.es ${ }^{2}$ o se puede acceder al canal de televisión del Ins-

${ }^{1}$ El Instituto Cervantes tiene varios portales especializados a cuya información se puede acceder desde el portal central http://www.cervantes.es, desde donde también se tiene acceso a los portales de cada uno de los centros del Instituto en el exterior.

${ }^{2}$ Para conocer en mayor detalle el proyecto se puede consultar Caro Barroso y Hita (2008). 
tituto a través de Internet en http://cervantestv.es, con una programación que día a día incorpora información audiovisual sobre los más variados aspectos de las artes plásticas, la música y la danza, literatura y pensamiento, lengua y enseñanza, informativos, documentales, etc.

El compromiso con toda la comunidad que conformamos alrededor del aprendizaje y enseñanza del español lleva a los portales web del Instituto a una constante renovación con el afán de apoyar a todos los profesores y estudiantes en su labor ${ }^{3}$. En los portales http://www.cervantes.es y http://cvc.cervantes.es hallarán los profesionales de la enseñanza de español información y recursos didácticos digitales que ayudarán a su formación y labor en el aula. Así, por mencionar algunos ejemplos, se encuentran desde la edición digital de actas de diferentes congresos, como el Congresos Internacionales de la Lengua Española (CILE) en http://www. cervantes.es/lengua_y_ensenanza/congresos_lengua_espanola/informacion.htm, o el Congreso Internacional de Español para Fines Especificos (CIEFE) en http:// cvc.cervantes.es/ensenanza/biblioteca_ele/ciefe/default.htm; hasta la consulta en pdf o HTML del Marco común europeo de referencia para las lenguas: aprendizaje, enseñanza, evaluación en http://cvc.cervantes.es/obref/marco/ o el Diccionario de términos clave de ELE en http://crc.cervantes.es/ensenanza/biblioteca_ele/diccio_ ele/default.htm.

\section{EL APRENDIZAJE Y LA ENSEÑANZA DEL ESPAÑOL CON LAS TECNOLOGÍAS DE LA INFORMACIÓN Y LA COMUNICACIÓN (TIC)}

El Aula Virtual de Español se empezó a diseñar hace ya una década, en ese momento se concibió el AVE 1.0 integrando herramientas de comunicación y materiales didácticos organizados en temas, que a su vez se subdividían en diez unidades o, lo que en su momento llamamos "sesiones", equivalentes a una hora más o menos de trabajo del estudiante interaccionando con el sistema y recibiendo feedback formativo. Metodológicamente seguimos un planteamiento comunicativo basado en el enfoque por tareas.

El Aula Virtual de Español, AVE en http://www.cervantes.es/ave, es un espacio de comunicación del que disfruta la comunidad de estudiantes y los profesionales de la enseñanza de español de las instituciones relacionados con el Instituto

\footnotetext{
${ }^{3}$ Tal vez algún visitante asiduo de las páginas web del Centro Virtual Cervantes o CVC, o del Instituto, pueda pensar que sería deseable una velocidad de publicación de materiales y renovación todavía mayor. No obstante, siendo esto una posibilidad factible, no hay que dejar de subestimar el tiempo que conlleva fraguar una idea en proyecto, y hacer de ésta una realidad editorial. El Instituto Cervantes es una institución creada en 1991 que comienza su andadura en 1992, en algo más de quince años se ha convertido en una institución de referencia en el ámbito que nos ocupa, la enseñanza de español.
} 
Cervantes. La plataforma AVE la concebimos como un espacio "integrador" y facilitador de intercambio de experiencias, en el que se provee al estudiante de servicios, materiales, seguimiento y, en definitiva, una serie de recursos con los que va a gestionar la construcción del conocimiento de la lengua y la cultura hispanas en comunicación con la comunidad de estudiantes de la que forme parte. Dos son los grandes protagonistas a los que dirigimos nuestra constante atención para evolucionar el soporte que se les ofrece, el alumno y el profesor.

El alumno, cuando introduce su contraseña, tiene frente a sí un escenario de trabajo en la "Sala de estudio" que

1. Proporciona un Material Didáctico Digital, MDD, y herramientas para que el aprendiente gestione su autonomía y responsabilidad respecto al proceso de aprendizaje.

2. Facilita y fomenta el intercambio de informaciones entre el grupo de estudiantes que aprende español y su tutor.

Respecto al aprendizaje centrado en el alumno y su manifestación a través del fomento de la responsabilidad y autonomía del estudiante, estamos convencidos de que a partir de las interacciones previstas con el sistema en el diseño multimedia del MDD y de la retroalimentación o feedback formativo que posibilita la tecnología, tenemos frente a nosotros una de las respuestas que, precisamente, permiten poner a disposición del estudiante una herramienta con la que podrá ejercitar la autonomía. Para ello, el diseño del material en "secuencias de aprendizaje lineal" junto al diseño de "material complementario" compuesto por actividades independientes que focalizan el objetivo lingüístico en ciertos aspectos de la lengua y la cultura hispana (ampliando una explicación o proponiendo una práctica más controlada), podría ser una de las claves para hacer realidad uno de los presupuestos que ya desde la década de los 70 se apuntaba como fundamental, al menos a nivel teórico: transferir al estudiante la responsabilidad que venía ejerciendo el profesor sobre el proceso de aprendizaje, para convertirse él en orientador de ese proceso ${ }^{4}$.

El profesor ejercerá su rol desde el escenario llamado "Gestión docente":

1. Un espacio para la formación en línea de los profesores: cursos de formación autónomo de tutores AVE, a modo de guía del tutor interactiva, construido con la intención de secuenciar al profesor el descubrimiento de todos los entresijos del AVE, e introducirlo en la reflexión sobre las modalidades de explotación en el AVE: apoyo de las TIC (Tecnologías de la Información y la Comunicación) a la enseñanza presencial, enseñanza

\footnotetext{
${ }^{4}$ Para más detalle sobre esta propuesta, conviene consultar la reflexión vertida en el reciente artículo titulado "La autonomía y el fomento de la responsabilidad del estudiante: los MDD (Materiales Didácticos Digitales) e Internet en el aula” de O. Juan Lázaro (2008).
} 
semipresencial y enseñanza a distancia.

2. Unas herramientas de comunicación que propician las posibilidades de interacción con sus otros colegas y les permite compartir nuevos usos y experiencias resultado de la constante evolución en la propuesta de inclusión de las TIC en la enseñanza de lenguas.

3. Un espacio propio para comunicarse con su grupo de estudiantes de español, en el que accede a herramientas de seguimiento de las actuaciones lingüísticas de cada estudiante, así como le ofrece la posibilidad de colgar material propio, facilitar instrucciones de actividades nuevas o acceso a enlaces de Internet, etc., para guiar y dar respuesta a las necesidades de su grupo de estudiantes.

\section{EL AULA VIRTUAL DE ESPAÑOL (AVE) Y LA WEB 2.0: LA EVOLUCIÓN EN EL DISEÑO Y CONCEPCIÓN HASTA LLEGAR AL PLANTEAMIENTO DEL AVE SOCIAL}

Hace cinco años se empezó a independizar lo que ahora es la plataforma de aprendizaje AVE 2.0 (Aula Virtual de Español) de los Materiales Didácticos Digitales (MDD) que conformaban los cursos de los diferentes niveles (del A1.1 al C1.4 del Marco europeo de referencia para las lenguas).

El brazo digital de la sociedad del siglo XXI extiende la evolución tecnológica a todos los ámbitos, incluidos, aunque tal vez de forma más tardía que en otros como el personal o el bancario, proyectos relacionados con la educación. Varias son las preguntas que nos planteamos y a las que intentamos responder tanto desde un punto de vista conceptual como desde la praxis, siendo conscientes de que la Plataforma AVE tiene que ser actualizada constantemente al igual que los materiales y cursos que integra, y por ende los modelos de enseñanza-aprendizaje de cuyo diseño forma parte (la enseñanza presencial que integra las TIC, la enseñanza semipresencial y la enseñanza a distancia). Estas son algunas de esos interrogantes que mantenemos en constante revisión:

1. Una reflexión constante sobre cómo se produce el aprendizaje y cómo ayudar a organizar dicho proceso de aprendizaje con las TIC.

2. Qué herramientas favorecen las interacciones comunicativas entre los miembros de la comunidad (el intercambio de información y la construcción del conocimiento).

3. Cómo diseñar los Materiales Didácticos Digitales, integrando soluciones de usabilidad así como tratando de fomentar la atención a la diversidad y respondiendo a las necesidades específicas de cada estudiante (nos referimos, concretando, por ejemplo, a que en un grupo vamos a encontrar 
a estudiantes que hayan previsto la dedicación lo más ajustada posible con el propósito de alcanzar los objetivos del curso, mientras que otros estudiantes tienen la necesidad de ampliar para sentirse seguros en lograr dichos objetivos).

4. Cómo favorecer la personalización de soluciones de aprendizaje adaptadas a cada centro, a cada país, a cada contexto geopolítico y cultural.

5. Cómo favorecemos que los profesores participen de la filosofía que subyace a la concepción de aprendizaje en el AVE.

6. Cómo vamos a seguir experimentando y repercutiendo los resultados en la actualización de cada una de los componentes del AVE.

Sobre el punto 1, seguimos las directrices generales que se recogen y postulan en el Common European Framework of Reference for Languages: Learning, Teaching, Assessment (Strasbourg: Council of Europe, 2001, en http://www.coe.int/), y cuya adaptación para el español corrió a cargo del Instituto Cervantes (http://cvc.cervantes.es/obref/marco/). De igual forma, es obvio que es imprescindible estar al corriente de las orientaciones que respecto a la educación emanan aires de innovación tecnológica, independientemente del continente del que provengan. En este momento hay que seguir de cerca los postulados metodológicos del socioconstructivismo y de lo que ya se empieza a denominar "conectivismo".

Respecto a las herramientas que favorecen la colaboración, nos hallamos en un buen momento tecnológico. Si se trata de fomentar las interacciones en la comunidad de estudiantes del AVE en la enseñanza semipresencial y en la enseñanza a distancia, nos encontramos con que la Web 2.0 implica compartir y comunicación, por lo que el enfoque orientado a la acción que se postula desde el Common European Framework encontrará total univocidad con los planteamientos de la Web Social. En este momento disponemos de más herramientas y servicios que podrían favorecer el intercambio espontáneo de los estudiantes y trasladar los usos sociales al ámbito educativo, acercándose a la realidad de los estudiantes en fronteras espaciotemporales que se diluyen con blogs, wikis, podcast, redes sociales, etc.

Precisamente tratando de identificar en los cambios que implican la Web Social o Web 2.0, y profundizar en qué consiste este "cambio de actitud" del usuario que pasa de ser lector a ser productor de contenidos y que se halla en la base de la Web Social, hemos realizado un estudio de cómo podría fraguarse la evolución del AVE actual al AVE SOCIAL, y en esto estamos trabajando y experimentando.

Para este estudio hemos trabajado junto con la Universidad de Alicante, con el grupo Virtua 5 adscrito al Departamento de Lenguajes y Sistemas Informáticos. Este sería un borrador de su posible aspecto, resultado de este estudio:

\footnotetext{
${ }^{5}$ Virtua, Centro de desarrollo de proyectos e-learning, en http://www.virtua.ua.es/.
} 


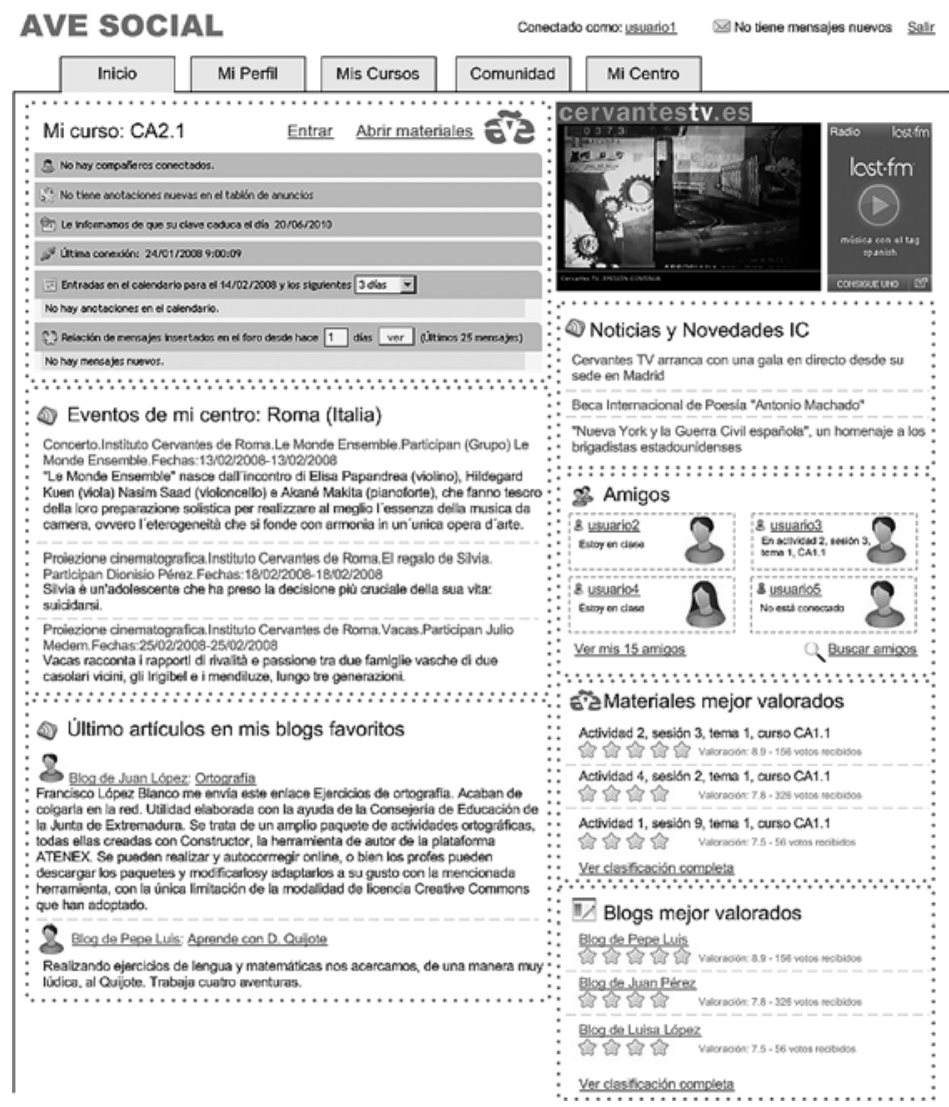

Figura 1. Aspecto del AVE Social concebido como un Entorno Personal de Aprendizaje. EPA (en inglés VLE, Virtual Learning Environment) en el que el estudiante se configura o sindica a los servicios y páginas web que le interesan.

La posibilidad de personalizar su Sala de estudio cada estudiante, incluyendo blogs, asociación por sindicación o RSS, "folcsonomías", etc., no suponen más que la participación comprometida y responsable del estudiante en su propio proceso de aprendizaje, ¿y acaso no es esta premisa de trabajo algo que tampoco es ajeno en la metodología comunicativa de enseñanza de lenguas en lo que estamos inmerso?

La Web 2.0 propicia los servicios que dan respuesta a una actitud de colaboración. Pero la respuesta de la sociedad ante el reto de la construcción del aprendizaje y las tecnologías, no avanza al mismo ritmo en el ámbito de la educación. Basta acercarse a los estudios sobre el impacto de las TIC para comprobar, por ejemplo, que todavía se está recorriendo el camino que conduce a la efectiva dotación de infraestructura en los centros y la contratación de un sistema de mantenimiento informático real, por no mencionar la reivindicación constante de una formación de los profesores adecuada a las habilidades que se les pide que promuevan en sus 
estudiantes, o la necesidad de disponer de repositorios de materiales de una calidad fiable. Estos resultados son evidenciados por informes como los de European Communities by European Schoolnet (2006), en España el estudio del Ministerio de Educación y Cultura y Ministerio de Industria, Turismo y Comercio (2006), junto con los que hace referencia M. Benito en su artículo (2008) sobre "Educar en comunidad: tendencias educativas en el nuevo entorno digital", mencionando los resultados de los estudios del NSBA en 2007 (Nacional School Board Association, en el que conformaron la muestra 1300 estudiantes entre 9 y 17 años, 1.000 padres y 250 directores de escuela) o el Estudio del Eurobarómetro, de mayo de 2007.

En los diferentes informes se pone de manifiesto desde el punto de vista del uso de la Web, en general, la familiaridad de los encuestados con Internet y el acceso desde el hogar con uso diario de una a tres horas en el caso de estudiantes entre 12 y 14 años, con el objetivo principalmente de buscar información y participar en juegos. En los grupos de más edad destaca el uso de los recursos de comunicación como chat y correo electrónico junto con la descarga de música, vídeos, etc. Menos frecuente es la creación de blogs o páginas personales. Según M. Benito, los resultados apuntan a poder afirmar que "los jóvenes están acostumbrados a usar Internet y sus recursos, si bien el uso de redes sociales no está totalmente generalizado" y añade que "las redes sociales pueden ser una poderosa herramienta de formación en el futuro", y nosotros nos preguntamos, cuando hablamos de futuro, ¿a qué momento nos estamos refiriendo? En la Sociedad Digital se han revolucionado las comunicaciones y el acceso a la información, y estas nuevas posibilidades de las TIC han traído consigo grandes cambios que aun hoy se siguen operando y que no todos somos capaces de vislumbrar, pero coincidiremos al afirmar los que tratamos de "estar al tanto" de los pasos de estos cambios en la era de la gestión del conocimiento que el tiempo corre en nuestra contra y el futuro es ese intangible que lo tenemos casi pululando como nuestra sombra. Normalmente, este tipo de encuestas e informes experimenta una curva creciente exponencial, lo que nos hace sospechar que hoy puede no estar extendido el uso de redes sociales, pero el "futuro" se vislumbra muy cercano, y mañana podemos estar frente a unas estadísticas que arrojen datos abrumadores sobre el uso de blogs, podcast, redes sociales, etc.

Sin embargo, a pesar de no ser halagüeños los resultados arrojados por los más recientes estudios para extender el uso de la tecnología como un elemento más indisociable del aula, la evolución a la que apuntamos con la creación del AVE SOCIAL responde a "facilitar" a los usuarios del AVE las herramientas y servicios de comunicación e interacción que permitirían que se llegase a conformar "la comunidad" de estudiantes y profesores de español que interactuarían y aportarían recursos relevantes a la experiencia de enseñanza y aprendizaje (por ejemplo, vídeos, fotos, material auditivo, referencias a eventos importantes... e incluso, 
las recomendaciones a otros compañeros sobre las actividades que más les han gustado, catalogadas u organizadas siguiendo los criterios generados en la propia comunidad).

Antes de llegar a conformar esta "comunidad en el AVE SOCIAL", parece desde el punto de vista tecnológico, así como conceptual, que tendría que producirse un paso intermedio. Los estudiantes y los profesores participan ya de sus propias redes sociales, en las que van sindicando e integrando los diferentes servicios de la Web Social, por lo que sería más idóneo plantearse qué utilidades, recursos y servicios del AVE actual podrían ser susceptibles de formar parte de esas redes. Es decir, qué servicios del AVE estuvieran accesibles en iGoogle o Facebook, según muestran los siguientes gráficos:

\section{Integración con otras plataformas}

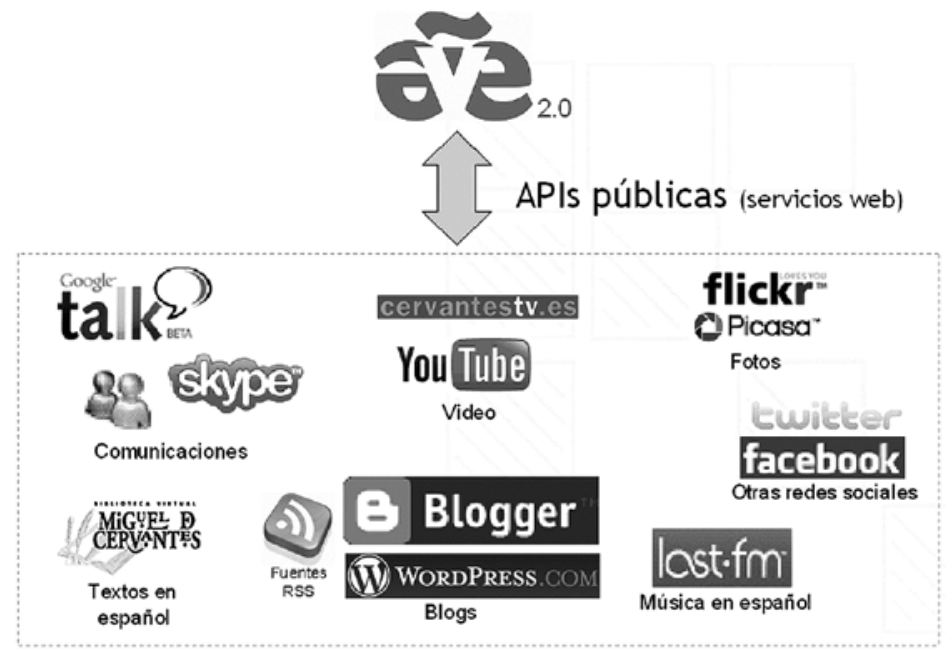

Figura 2. Ejemplo de integración del AVE 2.0 (que indica la versión del AVE actual) en los diferentes servicios de la WEB SOCIAL que aparecerían en el EVA integrados.

Esta fase en la que el AVE integra servicios de audioconferencia como los que de momento provee, por ejemplo, skype de forma gratuita, o videoconferencia está muy acorde a la práctica habitual entre nuestros profesores y estudiantes en la modalidad a distancia cien por cien en el AVE. La práctica de la interacción oral era, o todavía es, un escollo en el aprendizaje de lenguas online. Pero no nos hemos encontrado con el rechazo de ningún grupo todavía respecto a la integración de otros servicios que complementan las posibilidades de comunicación de la propia plataforma AVE, aunque es cierto, y hay que ensalzar la labor de los pro- 
fesores, es una gestión administrativa más el dar de alta a todo el grupo fuera del AVE y organizarlo todo para que funcione. De esta forma, lo que nos propicia los EPA (entornos personales de aprendizaje) es tener un escenario diseñado a medida que integra servicios y recursos de la web que hacen casi innecesario desarrollar soluciones de forma personalizada para cada plataforma.

\section{API pública: Herramienta para iGoogle}
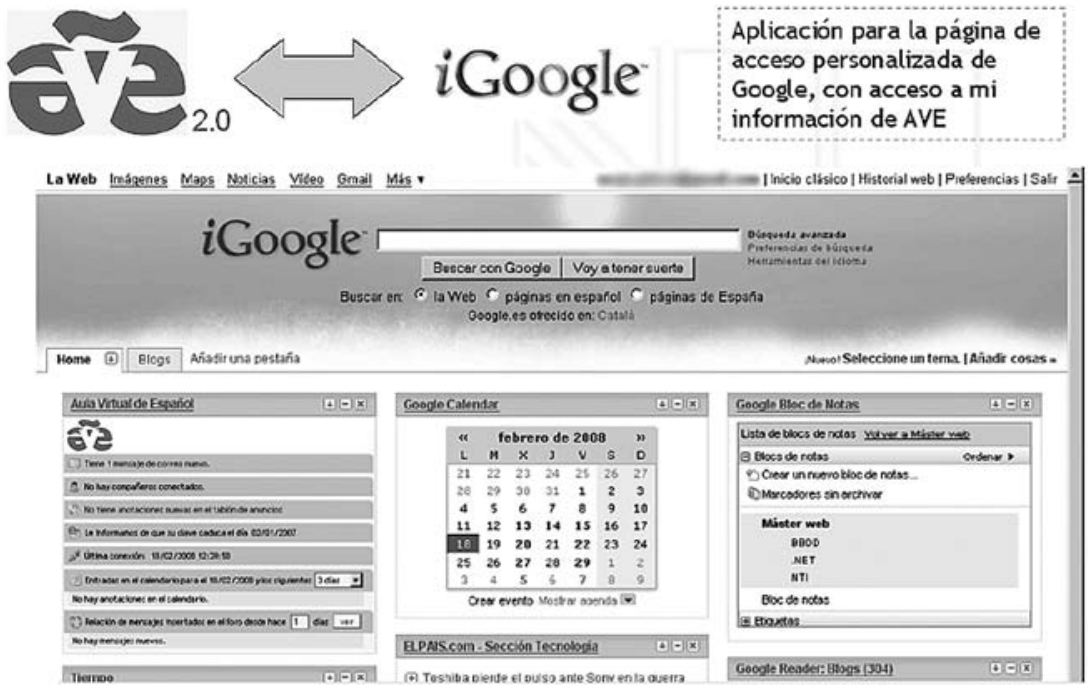

Figura 3. Cómo los diferentes servicios del AVE aparecerían formando parte, por ejemplo, de iGoogle, siendo este el entorno personal del estudiante.

La evolución al AVE SOCIAL implicaría, además de una apuesta por la experimentación constante con las TIC y su integración en el aprendizaje de español, un importante cambio conceptual que está en la propia génesis de la Web Social. En el histórico de usuarios del AVE del año 2007 (los últimos publicados) se hallan registrados 68.000 alumnos y 4.600 tutores, siendo los activos en ese año 16.000 alumnos y 1.000 tutores. En el caso de estar inmersos en la red del AVE SOCIAL estaríamos hablando de una comunidad potencial de 72.000 usuarios entre estudiantes y profesores. Esta comunidad está integrada, además, por instituciones de muy diversa índole, desde los estudiantes y equipos docentes de los centros del Instituto Cervantes distribuidos por el mundo (más de 70 en este momento), centros acreditados y asociados del Instituto Cervantes, universidades públicas y privadas, y empresas multinacionales que han optado por una formación en español a partir del AVE. 


\section{Comunidad de Usuarios}

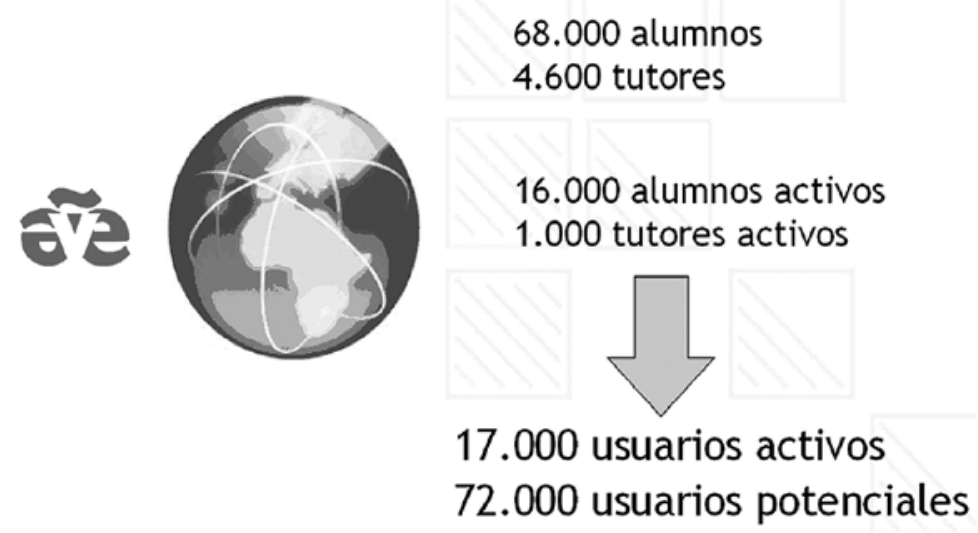

Figura 4. Ejemplificación del potencial de usuarios del AVE sumando los datos del histórico con los usuarios activos. Base de datos de la Administración del AVE 2007.

Desde el punto de vista del impacto en la educación de la Web 2.0, Anderson (2007) hace referencia al "efecto de la red" en el que las comunidades actúan de forma autosostenida y autónoma, una vez que los usuarios han percibido el beneficio de la participación y son conscientes de que el servicio mejora a medida que participan más en él. Este es un gran reto cuando se trata de propuestas cuya génesis está en el supuesto interés de los usuarios por conformar una red social en torno al aprendizaje del español y su enseñanza, y en nuestro caso concreto, en torno a la experiencia a partir del uso del AVE. Este tipo de reflexiones y debates van a estar siempre abiertos, obligándonos a todos los profesionales a tomar decisiones constantemente, por ejemplo, respecto a qué parte de los datos, información, ficheros, etc., tienen que ser generados por el Instituto Cervantes, y qué parte generado por la comunidad libremente pasando a formar parte de esa información finalmente compartida.

Los aspectos tecnológicos son una condición necesaria para que se evolucione en los modelos de enseñanza, aunque no son la clave (varios son los autores que destacan este matiz). La archiconocida cuestión de "qué hacer con la tecnología" surge una vez asegurados los recursos tecnológicos (Owen, 2006, y Evans, 2007, citados por M. Benito, 2008). En este artículo lo que estamos planteando es precisamente cómo racionalizar la evolución de los recursos y servicios dentro del AVE a partir de los datos objetivos existentes para seguir experimentando con los modelos de enseñanza. 


\section{LOS MATERIALES DIDÁCTICOS DIGITALES EN LA PLATAFORMA AVE: LA COMUNIDAD DE PROFESORES Y ESTUDIANTES}

Los Materiales Didácticos Digitales nos parece que tienen un protagonismo, en principio pasivo, que no conviene pasar por alto. Un buen diseño del material didáctico nos va a dotar de una perdurabilidad que desfocalizará la atención sobre el costoso proceso de edición (el trabajo de equipos multidisciplinares es necesario en este campo, exigiendo una alta coordinación y nivel de implicación de todos para sacar el máximo partido a las propuestas informáticas). En nuestro caso, las actualizaciones del material consisten en la corrección ortotipográfica que siempre se escapa (a pesar de contar con redactores y correctores especializados en el proceso de edición) y la sustitución de referencias a páginas web que hayan ido quedando obsoletas.

Los Materiales Didácticos Digitales (MDD) de los cursos se volvieron a programar informáticamente para independizar las actividades de la secuencia de trabajo lineal (compuestas de una a cuatro pantallas). Esto nos permite un escenario flexible en el que disponemos de aproximadamente 15.000 "objetos de aprendizaje", etiquetados de acuerdo al LOM (Learning Object Metadata) LMS, una de las catalogaciones que sigue el estándar SCORM internacional. El trasfondo que subyacía en esta actualización era posibilitar la formación de cursos e itinerarios en la enseñanza semipresencial y a distancia, por un lado, para dar respuesta a las necesidades de cada centro y, por otro, para facilitar y garantizar la complementariedad de los recursos tecnológicos a los ya usados en las clases presenciales. Se deja abierta la posibilidad de seleccionar actividades para conformar nuevas "sesiones" y "temas" según la programación de los centros que opten por el AVE.

También ligado a este uso, la reorganización flexible del MDD, nos encontramos con que se daba paso a la posibilidad de dar respuesta a contextos diferentes de los previstos inicialmente para el aprendizaje del español. Nos referimos, por ejemplo, a crear cursos a partir del material existente adaptados al currículo de español como primera lengua en la enseñanza reglada; o reorganizar el material y diseñar un nuevo curso para la enseñanza de español como lengua de integración para la enseñanza de adultos en el contexto pluricultural y plurilingüístico de la sociedad española actual; etc.

En la modalidad presencial, es decir, en la incorporación de las TIC a la clase presencial, el profesor identifica y reivindica desde el aula el rol que van a tener estos MDD dentro de la actividad programada para lograr los objetivos del curso.

Desde el constructivismo se habla de un estudiante responsable de su proceso de aprendizaje, lo cual implica que tiene que conocer su estilo de aprendizaje y las lagunas dentro para poder luego ejercitarse en ellas con más ahínco. Una vez que tiene esa consciencia, ¿cómo lo ejercita? Un material accesible en red, con un gran número de actividades (y en el AVE, para los cursos generales de español se 
cuentan más de 15.000 actividades entre los niveles A1.1 y C1.4), le va a permitir trabajar más con la comprensión audiovisual o hacer más énfasis en las actividades de morfología verbal, si así lo necesita, o en las propuestas para trabajar el vocabulario desde diferentes enfoques (mediante relaciones semánticas, con asociaciones a imágenes o a fragmentos de vídeo, etc.).

Para ello, una vez que el profesor lo ha introducido en clase, el estudiante tiene que estar capacitado para buscar y seleccionar lo que le conviene e interesa trabajar, y tener la posibilidad de compartir qué material le ha gustado más y qué material le ha sido más útil, y articular otra serie de posibilidades que impliquen compartir su experiencia. Esta es nuestra propuesta para integrar en el AVE SOCIAL, instrumentalizando las tendencias de la Web 2.0 al servicio del proceso de aprendizaje.

A continuación, reproducimos las portadas de dos de los cursos integrados en la plataforma AVE, destacando que cada uno de ellos tiene su propia identidad en cuanto a diseño y navegación (lo cual es posible debido a la independencia de la plataforma respecto a los MDD):

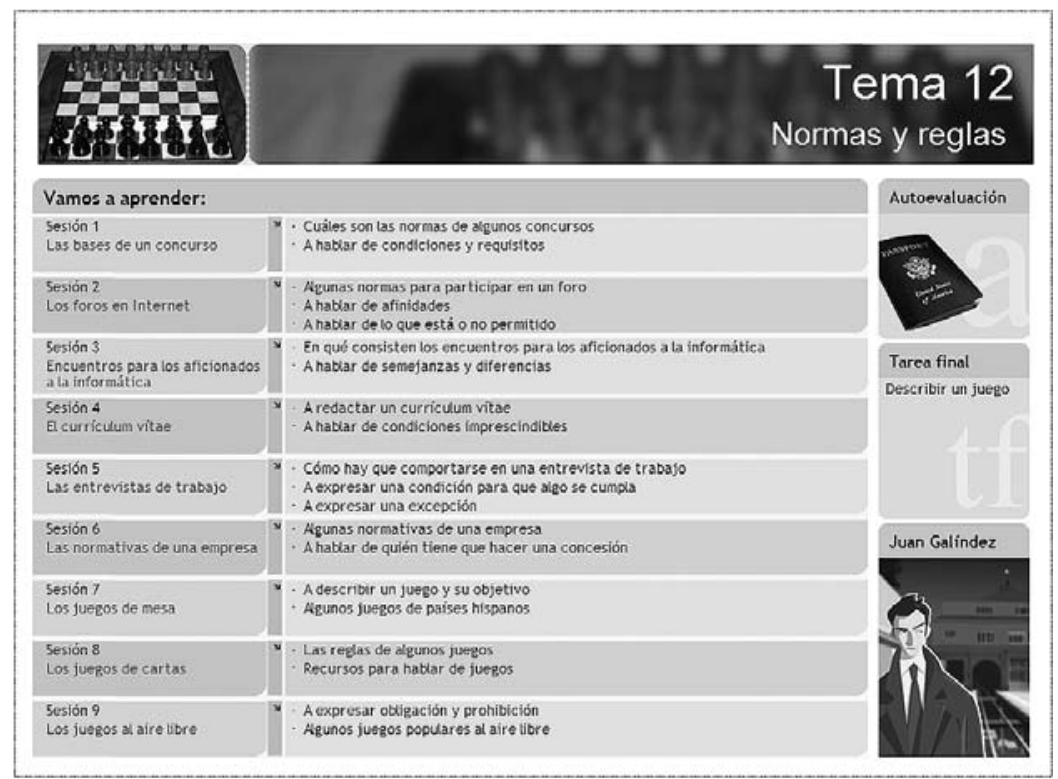

Figura 5. Portada del tema titulado "Normas y reglas" del nivel B2.4 del Curso general de Español, que trata sobre entrevistas de trabajo, los foros en Internet, los juegos al aire libre, etc., tratando de enfocar los objetivos lingüísticos del nivel a través de temas actuales y variados que ofrezcan en diferentes contextos los ítemes lingüísticos ${ }^{6}$.

${ }^{6}$ Para la descripción de los Cursos de español general del AVE, se puede consultar la información de la página web http://www.cervantes.es/ave/, seleccionando "Cursos de Español General", así como en los artículos de De Basterrechea y Juan Lázaro (2005); De Basterrechea (2004); Olalde Vegas y Juan Lázaro (2004); Juan Lázaro, O.; Duque, A. y Gil, M. (2002), entre otros. 


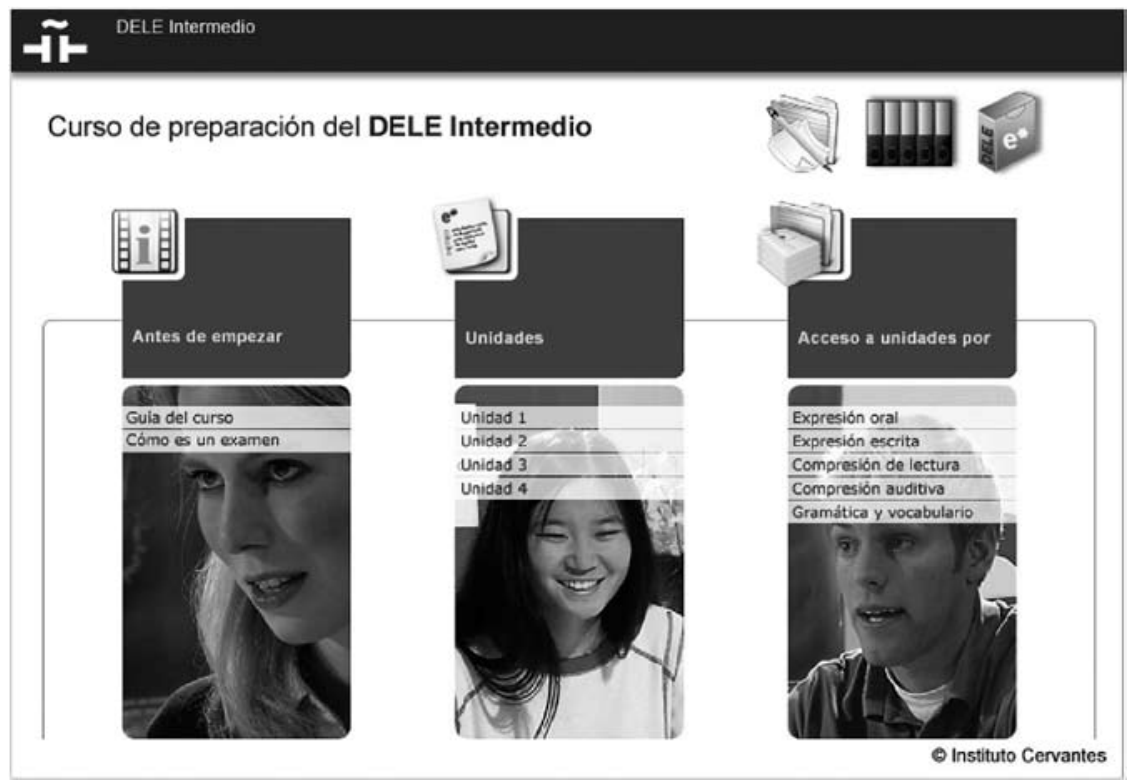

Figura 6. Portada del Curso de preparación al DELE Intermedio?

En este paso queremos hacer énfasis muy especial en los siguientes roles derivados del uso de las TIC en el aula de español, concretando la información que hemos venido detallando:

1. El profesor fomenta el uso de los MDD del Curso de español general, en nuestro caso, como prolongación del trabajo reflexivo y la interiorización de contenidos y procedimientos de comunicación con sus compañeros. El "Aula Tecnológica" es fundamental para ello, es decir, un aula en la que el profesor cuenta con un ordenador, un cañón de proyección y conexión a Internet (o Pizarra digital o interactiva) frente al "Aula Multimedia", es decir, un aula en la que se disponen de ordenadores suficientes para trabajar todos los estudiantes de una clase.

2. El profesor ayuda al estudiante a entender qué beneficios le va a reportar que él mismo tome las riendas de su proceso de aprendizaje, disfrutando y trabajando con aquellos aspectos de la lengua que son lagunas en su proceso de adquisición de la misma.

3. El estudiante se conecta allá donde lo necesita: hace el seguimiento de

${ }^{7}$ Para obtener más información sobre la estructura y las características del diseño instructivo seguidas en este curso recomendamos consultar Duque de la Torre, Aurora; Juan Lázaro, Olga y Milagros Ortín (2008), así como las demos de la página web http://www,cervantes.es/ave, seleccionando "Cursos de preparación del DELE". 
su aprendizaje y ejercita la lengua en los aspectos que cree (con índices o sistemas de búsqueda adaptados a su metalenguaje).

En el artículo de O. Juan Lázaro (2008) se definen los pasos para la efectiva integración de los MDD en el aula presencial, ejemplificándolo con los Cursos de Español General de la plataforma AVE; reproducimos aquí el gráfico:

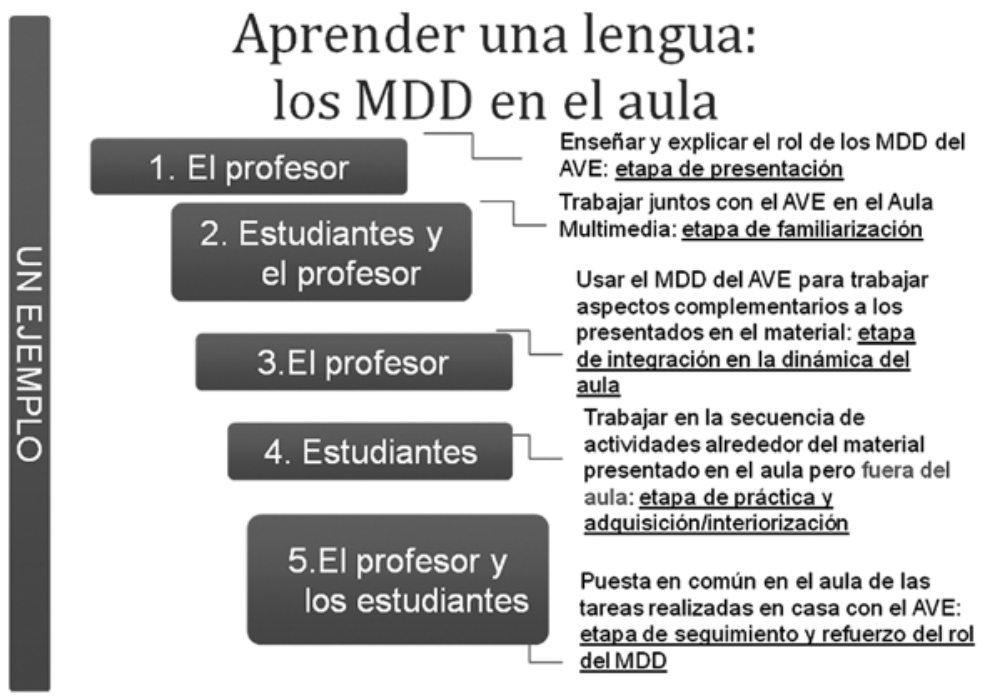

Figura 7. Propuesta de integración definida en etapas del MDD en Internet en la modalidad presencial (O. Juan Lázaro, 2008).

Este modelo parte de asumir en el aula el protagonismo de las dinámicas de interacción oral, potenciando el momento del cara a cara, ya que presuponemos el esfuerzo que para los estudiantes implica desplazarse hasta el centro en un horario concreto, por lo que hay que rentabilizar esos momentos de encuentro haciendo énfasis en el desarrollo de aquellas habilidades lingüísticas que fuera de ese escenario son más complejas de abordar. Por otro lado, no hay que olvidar que durante estos últimos años la gestión del "Aula Multimedia" se ha desvelado como compleja, tanto desde el punto de vista administrativo y económico, como a nivel académico. En el mismo artículo de O. Juan Lázaro (2008) se completa la visión de las fases de la integración de las TIC en el aula con una reflexión sobre el Aula Tecnológica vs. Aula Multimedia.

En la enseñanza semipresencial y a distancia apostamos por el aprendizaje en grupo con el apoyo de un tutor. Se trata de construir una comunidad con el interés común de aprender español a través de la diversidad de la cultura hispana. Si bien, 
tenemos que reconocer que también tenemos estudiantes autónomos que no están integrados en ningún grupo y que superan con su esfuerzo personal los objetivos del curso en el que están matriculados del AVE con éxito.

\section{CONSTRUCCIÓN DEL APRENDIZAJE: FOMENTO DE ACTITUDES COLABORATIVAS Y PARTICIPATIVAS EN EL APRENDIZAJE A DISTANCIA}

La experiencia de aprendizaje a distancia vamos a comentarla a partir de los recientes cursos de formación de tutores del AVE que hemos impartido desde el Instituto Cervantes, dentro del programa que se ofrece abierto al público desde el Departamento de Formación de la Dirección Académica.

Vamos a centrarnos en comentar esta experiencia considerando que se podría extrapolar a la experiencia que tienen los estudiantes de español en la modalidad a distancia. Por otro lado, con estos cursos cubrimos un ambicioso objetivo respecto a las demandas de nuestros propios profesores en el Instituto. Después de diferentes intentos de formación, y articulado el curso de "Formación autónoma de tutores del AVE" desde el lanzamiento del AVE al público (pretendiendo así atender a los más de 1.000 tutores del AVE de diferentes instituciones), la demanda seguía siendo "más formación". Analizando la situación y buscando respuestas, se decidió ofrecer formación en "inmersión", es decir, si se trata de que el profesor se ejercite en las mismas habilidades digitales que le vamos a pedir que promueva en sus estudiantes y fomente una actitud cooperativa entres ellos, lo idóneo es que las "experimente" en el mismo escenario de trabajo ${ }^{8}$.

El "Curso de Tutores del AVE a distancia" ha sido diseñado con el apoyo tutorial de varios jefes de estudio y profesores de los Institutos Cervantes que aportan la experiencia de los cursos que han impartido en modalidad a distancia y semipresencial con el AVE tutorizados durante estos últimos cuatro años (en este caso Berlín y Frankfurt, Mánchester, Sao Paulo). Junto a ellos, son también tutores los técnicos que forman parte de la Unidad de Nuevas Tecnologías, Área Académica del Instituto Cervantes, donde se ha concebido, diseñado y gestionado la creación del AVE. De esta forma se combina el expertizaje de todos los profesionales que tienen relación con el AVE para ofrecer una panorámica amplia y a la vez detallada de todo lo relacionado con los planteamientos y trabajo de campo del AVE. Este curso de formación a distancia ha tenido dos ediciones durante el año 2008 y una edición durante el 2009. Actualmente, con los resultados de esta experiencia

${ }^{8}$ G. Salmon (2000) de la Open University lleva ya una década formando a tutores online en estas habilidades y requerimientos del entorno cien por cien a distancia. En Provencio Garrigós y Juan Lázaro (2005) se describen las funciones de un tutor online. 
estamos ya trabajando en la especificación de la actualización de la estructura y contenidos del curso.

Este es el aspecto de la entrada a la Gestión Docente con el curso de formación de tutores desplegado, al que todos los profesores que tienen usuario y contraseña del AVE tienen acceso:

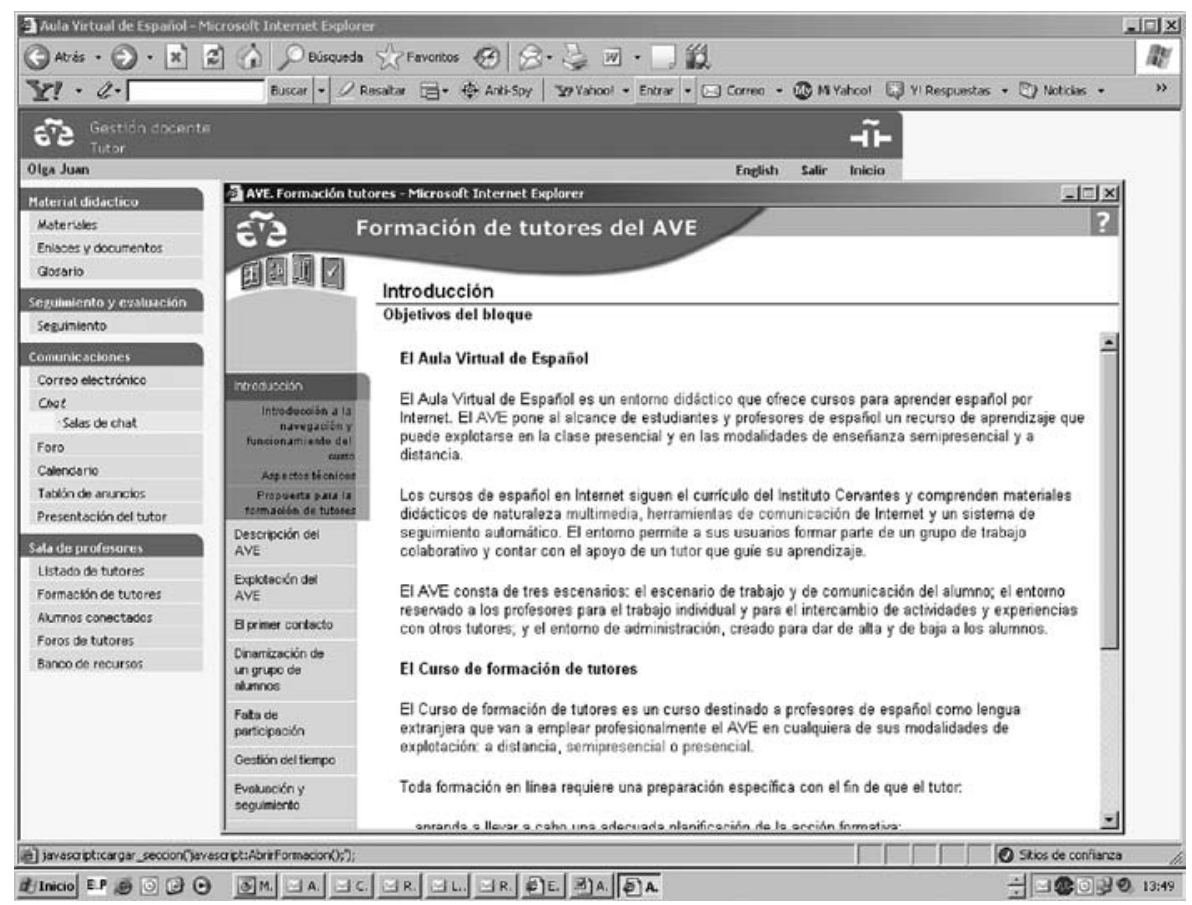

Figura 8. Pantalla de introducción del Curso de Formación de Tutores del AVE autónomo, dentro del escenario de "Gestión docente" donde trabaja el tutor.

En el diseño del curso con tutores se trabajó con dinámicas de actividades que llevan a la reflexión constante de los profesores, basándose en las aportaciones de los tutores en foros y de bibliografía y preguntas dirigidas al debate. He aquí algunos de los comentarios de la comunidad de profesores o tutores que formamos en la plataforma del AVE, en la modalidad de enseñanza a distancia. Son ejemplos ilustrativos del valor de la experiencia de los compañeros en la enseñanza a distancia, y del trabajo del tutor como orientador y facilitador del proceso de construcción de conocimientos. 


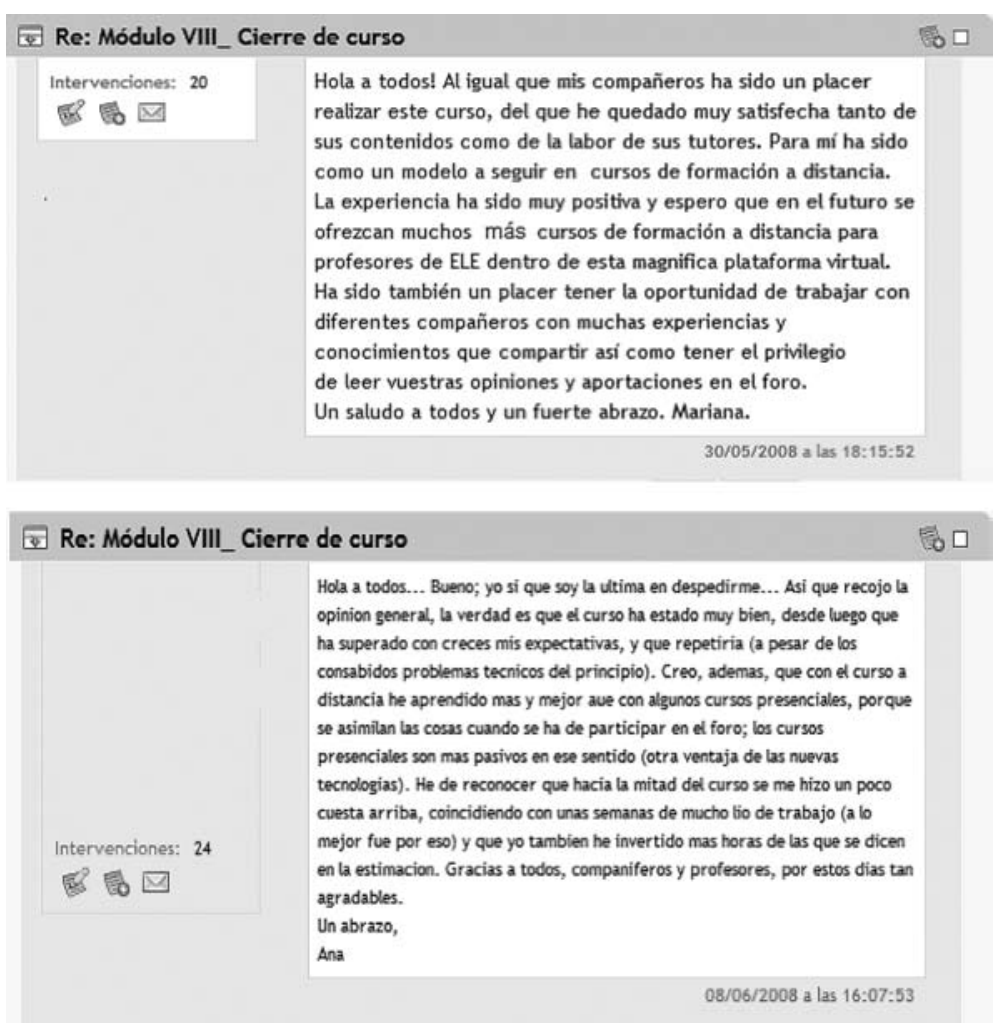

Figura 9. Comentarios de dos profesores al finalizar el curso de "Formación de Tutores del AVE a distancia".

De estos comentarios, lo más relevante bajo nuestro punto de vista es leer: "ha sido un placer tener la oportunidad de trabajar con diferentes compañeros con muchas experiencias y conocimientos que compartir así como tener el privilegio de leer vuestras opiniones y aportaciones en el foro", y también "con un curso a distancia he aprendido más y mejor aún que con algunos cursos presenciales, porque se asimilan las cosas cuando se ha de participar en el foro; los cursos presenciales son más pasivos en este sentido (otra ventaja de las nuevas tecnologías)".

En la base metodológica del planteamiento para llevar a cabo este curso se encuentra el socioconstructivismo ${ }^{9}$, el aprendizaje colaborativo ${ }^{10}$ y el aprendizaje en

9 Para revisar brevemente el concepto recomendamos buscar "Enfoque socioconstructivista" en http://es.wikipedia.org/wiki/Constructivismo_(pedagog\%C3\%ADa) y el Diccionario ELE del CVC, Constructivismo en http://cvc.cervantes.es/ensenanza/diccio_ele/indice.htm\#c.

${ }^{10}$ Recomendamos consultar para hacernos una idea de forma rápida "Aprendizaje colaborativo"

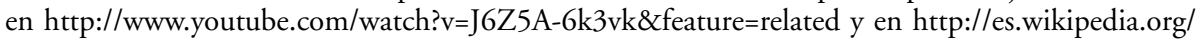
wiki/Aprendizaje_colaborativo. De forma complementaria, recomendamos "Aprendizaje autónomo" en http://cvc.cervantes.es/ensenanza/diccio_ele/indice.htm\#c. 
comunidades ${ }^{11}$, donde el reto se encuentra en proponer dinámicas de actividades y agrupaciones en las que los estudiantes aprecien el valor que aporta el bagaje de conocimientos y experiencias de los compañeros, y como hemos comprobado, independientemente de las herramientas usadas para el trabajo colaborativo que son simplemente medios.

\section{CONCLUSIONES}

En resumen, los 16.000 estudiantes que en el año 2007 se registraron en la administración del AVE, y los 1.000 tutores repartidos en las 150 instituciones que utilizan el AVE, nos implican un compromiso con la evolución tecnológica constante. La enseñanza semipresencial y a distancia nos encaminan a la propuesta del AVE SOCIAL en la que estamos trabajando, y esto involucra actualizaciones en los servicios y opciones de participación que permite la plataforma, y que abordamos en paralelo al fomento del cambio de actitud en la enseñanza a distancia, para poder así también rentabilizar las actualizaciones informáticas que sufren los servicios en red, en Internet, y que conllevan las propuestas del socioconstructivismo y la WEB 2.0. En este punto, tenemos que ser conscientes del binomio que se tiene que dar en la percepción del usuario: participación versus aprovechamiento.

Respecto a la incorporación de las TIC en la enseñanza presencial, el AVE promueve el acceso al MDD de forma personalizada, a partir del fomento de su uso por parte del profesor en el aula, y del fomento de una actitud responsable del estudiante hacia su proceso de aprendizaje, poniendo a disposición unos materiales secuenciados e indexados con los que podrá ejercer su autonomía. Pautar y guiar el itinerario de aprendizaje del estudiante es necesario, destacando desde este punto de vista la aplicación del diseño instructivo para potenciar el rol de los MDD y la autonomía del aprendiente.

Y sobre la enseñanza a distancia, la experiencia tanto con estudiantes como con formación de profesores nos lleva a poder afirmar que, efectivamente, las herramientas seleccionadas tienen un carácter esencialmente mediador, ya que es la propia comunidad que conforman profesores y estudiantes la que tiene que experimentar cómo se llega a la construcción del proceso de aprendizaje a partir del trabajo cooperativo, con la experiencia del beneficio de este trabajo en grupo, es decir, beneficiándose del socioconstructivismo.

Si reflexionamos sobre la situación actual y su modelo de evolución nos lleva a sumarnos a quienes afirman que el desarrollo del componente tecnológico o la dotación informática son un primer paso inexcusable en la evolución del modelo

${ }^{11}$ Aprendizaje en comunidades en http://www.dreig.eu/caparazon/2008/08/15/foc08-1-delgrupo-a-la-comunidad-principios-basicos/ 
educativo en cualquiera de sus manifestaciones (desde la presencialidad a la distancia cien por cien, pasando por todos los modelos intermedios que configuran la enseñanza semipresencial).

\section{REFERENCIAS}

Anderson, Paul. 2007. What is Web 2.0.? Ideas, technologies and implications for education. Bristol: JISC. [En línea]. Disponible en: http://www.jisc.ac.uk/ Home/publications/publications/twweb2.aspx. Consulta: 30/09/2009.

Balanskat, Anja; Blamire, Rogery Stella Kefala. 2006. The ICT Impact Report. A review of studies of ICT impact on schools in Europe. [En línea]. Disponible en: http:// ec.europa.eu/education/doc/reports/doc/ictimpact.pdf o desde http://ec.europa. eu/education/programmes/llp/impact_en.html. Consulta: 30/09/2009.

Benito Gómez, Manuel. 2008. "Educar en comunidad: tendencias educativas en el nuevo entorno digital", en revista BIT 167.

Caro Barroso, José Carlos y Hita, Germán. 2008, "El Instituto Cervantes en Second Life", Actas del I Congreso Metaversos. Web 3D y Redes Sociales en Mundos Virtuales, Ibiza, 20-22/07/08. [En línea]. Disponible en: http://metaversos.com/2008/ponencias_2008. Consulta: 30/09/2009.

Consejo "Educación" de la Unión Europea. 2001. "Futuros objetivos precisos de los sistemas de educación y formación”. [En línea]. Disponible en: http://europa. en.int/comm/education/policies/2010/et-2010-html. Consulta: 30/09/2009.

CRUE. 2004. Las Tecnologías de la Información y la Comunicación en el Sistema Universitario Español, dirigido por S. Barro Ameneiro. CRUE, Conferencia de Rectores de Universidades Españolas. [En línea]. Disponible en: http:// www.crue.org. Consulta: 30/09/2009.

De Basterrechea, Juan Pedro. 2004. "Integración de los recursos didácticos digitales en la enseñanza del español. Hacia un nuevo paradigma", Identidad lingüística y globalización, el III Congreso Internacional de la Lengua Española, CILE, coorganizado por la Real Academia Española y el Instituto Cervantes en Rosario, Argentina. [En línea]. Disponible en: http://congresosdelalengua.es/ rosario/ponencias/internacional/basterrechea_j.htm. Consulta: 30/09/2009.

De Basterrechea, Juan Pedro y Juan Lázaro, Olga. 2005. "Influencia de los recursos digitales y los sistemas de comunicación en el modelo de enseñanza de ELE”, en Actas del Primer Congreso Internacional de FIAPE, Federación Internacional de Asociaciones de Profesores de Español. [En línea]. Disponible en: www. sgci.mec.es/redele/biblioteca2005/fiape.shtml. Consulta: 30/09/2009.

Duque de la Torre, Aurora; Juan Lázaro, Olga y Milagros Ortín. 2008. Integración de recursos digitales en la enseñanza de español: Curso de preparación del Diploma DELE. Actas del congreso internacional Virtual Educa. [En línea]. Dispo- 
nible en: http://www.virtualeduca.info/ponencias/101/DELE_VIRTUAL\%20 EDUCA_ZGZ_08.doc y desde http://www.cervantes.es/lengua_y_ensenanza/ aprender_espanol/cursos_dele_internet.htm. Consulta: 30/09/2009.

Evans, V. 2007. Networks, connections and community: learning with social software. Brisbaine: Australian Flexible Learning Framework.

Juan Lázaro, Olga; Duque de la Torre, Aurora y Gil, María. 2002. Actividades cooperativas a través de Internet: Cursos de Español a distancia del Instituto Cervantes. Online Educa Barcelona, 2a Conferencia Internacional de la Educación y de la Formación basada en las Tecnologías. Barcelona: ICWE y Universitat Oberta de Catalunya.

Juan Lázaro, Olga. 2008. Working on the Platform AVE of the Instituto Cervantes. 14th International Conference on Technology Supported Learning and Training, Online-Educa Berlin, December 3-5, 2008. [En línea]. Disponible en: http://www.online-educa.com/. Consulta: 30/09/2009.

Juan Lázaro, Olga. 2008 "La autonomía y el fomento de la responsabilidad del estudiante: los MDD (Materiales Didácticos Digitales) e Internet en el aula”.

Juan Lázaro, Olga. 2004. "Aprender español a través de Internet: un entorno de enseñanza y aprendizaje”, en Sánchez Lobato e I. Santos Gargallo (eds.), Vademécum para la formación de profesores. Enseñar español como segunda lengua y lengua extranjera. Madrid: SGEL, pp. 1087-1108.

MEC y Ministerio de Industria, Turismo y Comercio. 2006. Las Tecnologías de la Información y la Comunicación en la Educación. Informe sobre la implantación y el uso de las TIC en los centros docentes de primaria y secundaria (Curso 2005-2006). [En línea]. Disponible en: http://www.cnice.mec.es y http:// www.red.es. Consulta: 30/09/2009.

Ministerio de Educación, Cultura y Deporte; Instituto Cervantes. 2002. Marco común europeo de referencia para las lenguas: aprendizaje, enseñanza, evaluación, Ministerio de Educación, Cultura y Deporte, Subdirección General de Cooperación Internacional, Instituto Cervantes, Anaya, Madrid. [En línea]. Disponible en: http://cvc.cervantes.es/obref/marco/. Consulta: 30/09/2009.

Olalde Vegas, Silvia y Juan Lázaro, Olga. 2004. "Distance-learning Spanish courses: a follow-up and assessment system", en Studies in Language Testing 18, European Language Testing in a Global Context. Cambridge University Press, pp. 221-236.

Owen, M.; Grant, L.; Sayers, S. \& Facer, K. 2006. Social Software and Learning. Bristol U.K.: Future Lab.

Provencio Garrigós, Herminia y Juan Lázaro, Olga. 2005. Funciones del tutor y compromisos del alumno en la docencia semipresencial y a distancia de español (E) LE) Frecuencia-L. Madrid: Editorial Edinumen.

Salmon, Gilly. 2000. E-Moderating. The key to teaching and learning online. Londres: Ed. Kogan Page. La presentación del libro se puede consultar en: http:// www.atimod.com/e-moderating/extracts.htm. 